\title{
Atypical presentation of Schimmelpenning- Feuerstein-Mims syndrome associated to a giant congenital nevus: a one case report
}

\author{
Nadia Baali', Fatima Zahra Dyabi ${ }^{2}$, Fatiha Bennaoui ${ }^{2}$, Nadia El Idrissi Slitine ${ }^{2}$, \\ FadI Mrabih Rabbou Maoulainine ${ }^{2}$, Said Amal $^{2}$, Ouafa Hocar ${ }^{2}$
}

${ }^{1}$ Dermatology Department, Mohamed VI University Hospital, Marrakesh, Morroco, ${ }^{2}$ The Neonatal Intensive Care Unit, Mohamed VI University Hospital, Marrakesh, Morroco

Corresponding author: Dr. Nadia Baali, E-mail: nadia.baali@outlook.fr

\begin{abstract}
The Schimmelpenning-Feuerstein-Mims (SFM) syndrome is a congenital neurocutaneous disorder characterized by the association of sebaceous hamartoma with extracutaneous abnormalities. We report a case of an SFM syndrome associated with a giant congenital nevus in the neonatal intensive care unit (NICU) of Mohamed VI University Hospital of Marrakesh. This case has the specificity of being the first case reported in the literature associating an SFM syndrome with a giant congenital nevus. In conclusion, this observation is exceptional; it's a combination of the SFM syndrome to a giant congenital nevus, which so far has never been reported in the literature.
\end{abstract}

Key words: Schimmelpenning-Feuerstein-Mims syndrome; Giant congenital nevus

\section{INTRODUCTION}

Schimmelpenning-Feuerstein-Mims (SFM) syndrome is a rare congenital neurocutaneous disorder characterized by the association of sebaceous hamartoma with extracutaneous abnormalities in different neuroectodermal organ systems.

Its association with a giant congenital nevus has never been reported so far. We report the first case of an SFM syndrome associated with a giant congenital nevus in the NICU of Mohamed VI University Hospital of Marrakesh.

\section{CASE REPORT}

We report a case of a term male newborn, born by vaginal delivery, from a first degree consanguineous marriage, his mother aged 22-years, had 2 gestity, 2 parity, 2 children alive, the older brother aged one and a half years old with no reported abnormalities.
Admitted in his first day of life to the NICU; the newborn suffered from respiratory distress and neonatal infection.

The clinical examination revealed a newborn, reactive, with an absent sucking reflex, hypotonia, hepatomegaly and moderate ascites, respiratory distress rated at 4/10 according to the Silverman score and ascites of average abundance. The dermatological examination revealed a multiple yellow-orange lesions with a warty surfaces of geographical shapes on the scalp, the face and the neck evoking the sebaceous hematoma, in addition to a giant melanocytic congenital nevus which was extended to the entire body sparing the hands and feet, surmounted by medium-abundance hairiness associated with contact bleeding nodules, sebaceous scalp and facial hamartomas, and a large pilot liparus nevus (Figs. 1 and 2). The ophthalmological examination revealed eyelid swelling, conjunctivitis, corneal infiltrate in the left eye and corneal ulcer in the right eye (Fig. 3). The transfontanellar ultrasound

\footnotetext{
How to cite this article: Baali N, Dyabi FZ, Bennaoui F, El Idrissi Slitine N, Maoulainine FMR, Amal S, Hocar O. Atypical presentation of SchimmelpenningFeuerstein-Mims syndrome associated to a giant congenital nevus: a one case report. Our Dermatol Online. 2020;11(e):e109.1-e109.4.

Submission: 15.09.2019; Acceptance: 21.11.2019

DOI:10.7241/ourd.2020e.109
} 


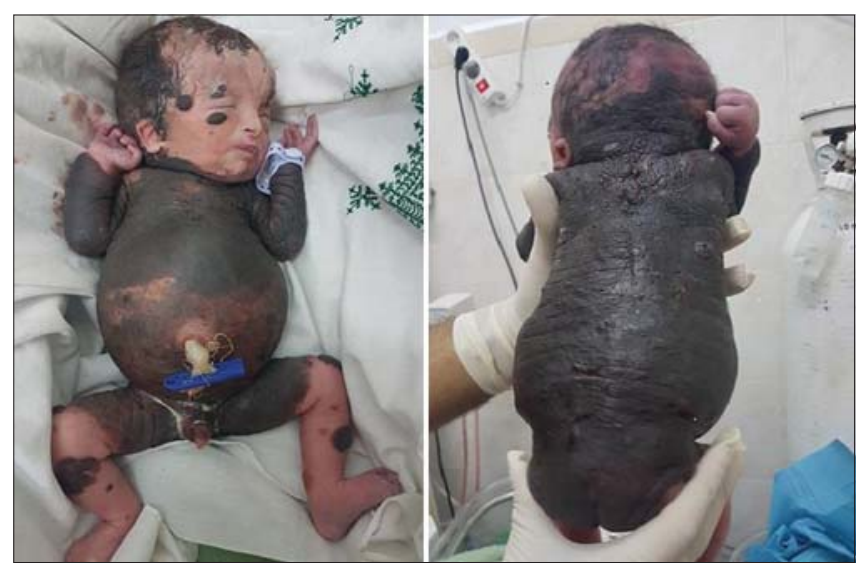

Figure 1: A giant melanocytic congenital nevus extended to the entire body sparing the hands and feet associated with contact bleeding nodules.

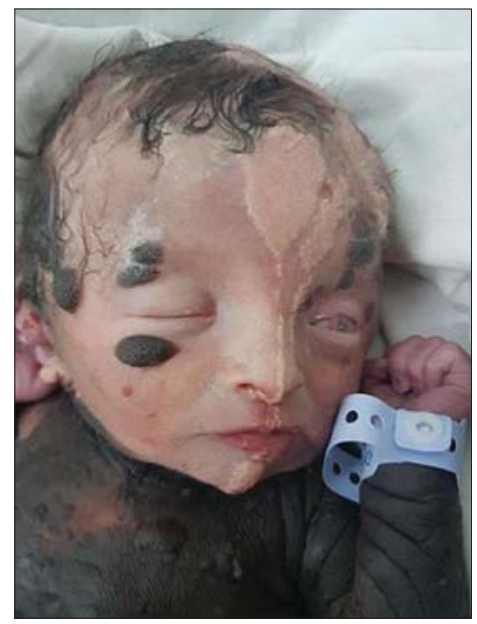

Figure 2: Sebaceous scalp and facial hamartomas.

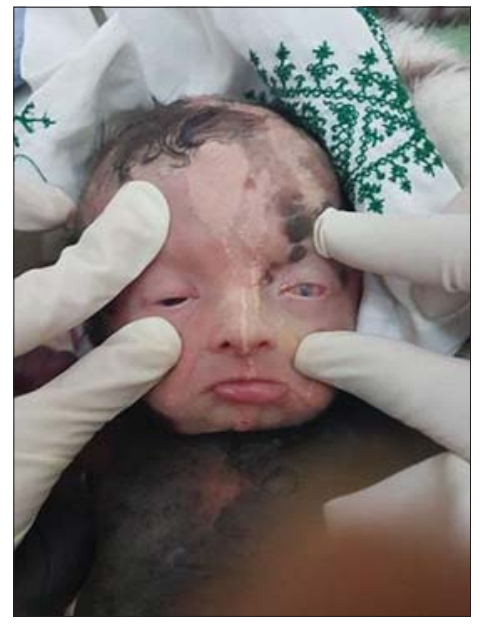

Figure 3: Eyelid swelling and conjunctivitis.

showed the hyperechogenicity of the thalamus and multiple cysts, especially in the interhemispheric area.

Cardiac ultrasound revealed a persistent arterial duct, with two ostium Secundum atrial septal defects. The anatomopathological study of hamartomas found a morphological aspect compatible with sebaceous hamartomas.

The neonate was initially treated by non-invasive ventilation and bi-antibiotic for the maternal-fetal infection, which was complicated secondarily by a nosocomial infection with multi-resistant Klebsiella pneumonia.

The death occurred at his twenty-fifth day of hospitalization, caused by uncontrolled severe sepsis.

\section{DISCUSSION}

The newborn presented with SFM syndrome, also known as a linear sebaceous nevus syndrome, a sporadic disease characterized by the association of sebaceous nevi and other cutaneous, neurological, cardiovascular and ocular abnormalities [1].

The etiology of this syndrome is still unknown, but the hypothesis of genomic mosaicism was emphasized [2]. This syndrome was first described in 1957 by Gustav Schimmelpenning who had reported a case associating a sebaceous hamartoma and neurological abnormalities [3].

A few years later, in 1962, Feuerstein and Mims reported a case of linear nevus associated with epilepsy and mental retardation defining the classic triad that associates sebaceous hamartoma, epileptic seizures and intellectual disability [4]. Several studies have found that SFM-related abnormalities extend well beyond the initial triad, resulting in the abandonment of all the diagnostic criteria. The sebaceous nevus, present in about $0.3 \%$ of births, is a feature of SFM [2]. Cutaneous involvement is characteristic of the syndrome [5], the presence of organoid nevi is necessary to make the diagnosis. It comes in the form of a yellow-orange, pink lesion with finely papillomatous o an alopecia lesion that can be oval or blaschkolineal [2].

In this observation, the clinical presentation of the sebaceous nevus was typical. The scalp is the most common location of sebaceous nevi (42\%), followed bythe face (32.6\%); then the periauricular region (3.8\%) then the neck $(3.2 \%)[1,5]$.

Neurological abnormalities occur in $66 \%$ of cases, which is the leading cause of morbidity and mortality for these patients. They can manifest as structural 
abnormalities (72\%), mental and cognitive impairment, convulsions, corpus callosum agenesis, DandyWalker malformation, Hemi megalocephaly and/or hemiparesis [6]. Neurological disorders were present in this case as excessive hypotonia and absent sucking reflex, hence the transfontanellar ultrasound showed hyperechogenicity of the thalamus and multiple cysts, especially in the interhemispheric area. However, additional magnetic resonance imaging was not done due to the hemodynamic and respiratory instability of the patient.

Ophthalmological abnormalities are present in $59 \%$ of SFM syndrome cases [6]. The main ocular abnormalities include strabismus, lipodermoid, and coloboma; therefore, blindness can occur [7]. Ophthalmological examination in this patient revealed eyelid swelling, conjunctivitis, corneal infiltrate in the left eye and corneal ulcer in the right eye.

Cardiovascular abnormalities present in $12 \%$ of cases include persistence of the arterial duct, patent foramen oval, coarctation of the aorta or hypoplasia of the aorta [6]. In this patient, persistent arterial duct, with two Ostia Secundum atrial septal defects were present.

The differential diagnosis includes other syndromes with cutaneous and neurological involvement, such as pigmentokeratica phacomatosis, nevus comedonicus syndrome and Proteus syndrome. In our patient, the diagnosis was focused on the clinical and anatomopathological features of the cutaneous lesions and the visceral abnormalities.

The management of these patients should be multidisciplinary. The prognosis will depend on the severity of the organic damage. The goal of management is to improve the quality of life of the patient. It is to notice that there is no cure $[7,8]$.

The association of SFM syndrome with giant congenital nevi has never been reported in the literature.

Giant congenital nevi are rare, and their incidence is estimated at 2/10 000 births [9]. These lesions because of their size, their pigmentation, sometimes hairy or nodular appearance, are responsible for real aesthetic damage.

It is most often located at the cervicofacial sphere then the trunk, extremities and more rarely on the abdomen. The risk of transformation into malignant melanoma varies between 1 and $15 \%$ but remains difficult to quantify exactly [10].

The pathophysiology remains unknown. Large naevi, however, are known to have a particular molecular signature through the presence of N-RAS mutations [11]. In this patient, no genetic test was performed.

There is no consensus on the management, and the therapeutic strategy is still not well established $[12,13]$ and should be discussed on a case-by-case basis for each patient, at best in a multidisciplinary consultation including pediatric dermatologists, surgeons, neuroradiologists, psychologists and taking into account the opinion of parents.

\section{CONCLUSION}

The Schimmelpenning-Feuerstein-Mims syndrome is a rare and variable multisystem defect. The combination of the SFM syndrome to a giant congenital nevus has never been reported in the literature. This case reports this rare association and highlights the importance of exploring patients with nevus sebaceous to diagnose associated anomalies.

\section{Consent}

The examination of the patient was conducted according to the Declaration of Helsinki principles.

\section{REFERENCES}

1. Resende C, Araujo C, Vieira APVentura F, Brito C. Schimmelpenning syndrome. Dermatol Online J. 2013;19:20026.

2. Bahloula E, Abid L, Masmoudi A, Makni S, Kamoun F, Boudawara T, et al. Le syndrome de Schimmelpenning-FeuersteinMims: à propos d'un cas. Arch Péd. 2015;221157-62.

3. Schimmelpenning GW. Clinical contribution to symptomatology of phacomatosis. Fortschr Geb Rontgenstr Nuklearmed. 1957;87:716e20.

4. Feuerstein RC, Mims LC. Linear nevus sebaceous with convulsions and mental retardation. Am J Dis Child. 1962;104:675e9.

5. Wang S, Hsieh Y, Chang K, Tsai H, Chen C. Schimmelpenning syndrome: A case report and literature review. Pediatr Neonatol. 2014;55:487-90.

6. Morales LK. Piel (Barc). Schimmelpennig Syndrome. 021392512016 Elsevier Espana2016.

7. Rabley A, Bayne CE, Shenoy A, DeMarco RT. Genital neurofibromatosis presenting as painful clitoromegaly. Urology. 2019;133:219-21.

8. Garcias-Ladaria J, Cuadrado Rosón M, Pascual-López M. Epidermal Nevi and related syndromes -- Part 1: keratinocytic nevi. Actas Dermosifiliogr. 2018;109:677-86.

9. Achehboune K, Zinoune S, Baybay H, Elloudi S, Mernissi FZ. Giant congenital nevus: About 3 observations. Our Dermatol Online. 
www.odermatol.com

2020;11:e47.1-e47.3.

10. Gatibelza ME, Denis D, Bardot J, Casanova D, Degardin N. Place actuelle du curetage dans la prise en charge des nævi géants congénitaux: à propos de 29 cas. Ann Chir Plast Esthet. 2013;58:228-34.

11. Shakhova O, Zingg D, Schaefer SM, Hari L, Civenni G, Blunschi J, et al. Sox10 promotes the formation and maintenance of giant congenital naevi and melanoma. Nature Cell Biol.2012;14:882-90.

12. Lawrence CM. Treatment options for giant congenital naevi. An Bras Dermatol. 2013;88:863-78.
13. Chaoui R, Baybay H, El Kadiri S, Achehboune K, Douhi Z, Elloudi S, Mernissi FZ. Giant congenital melanocytic nevus associated with lipoma. Our Dermatol Online. 2020;11:e7.1-e7.3..

Copyright by Nadia Baali, et al. This is an open-access article distributed under the terms of the Creative Commons Attribution License, which permits unrestricted use, distribution, and reproduction in any medium, provided the original author and source are credited.

Source of Support: Nil, Conflict of Interest: None declared. 